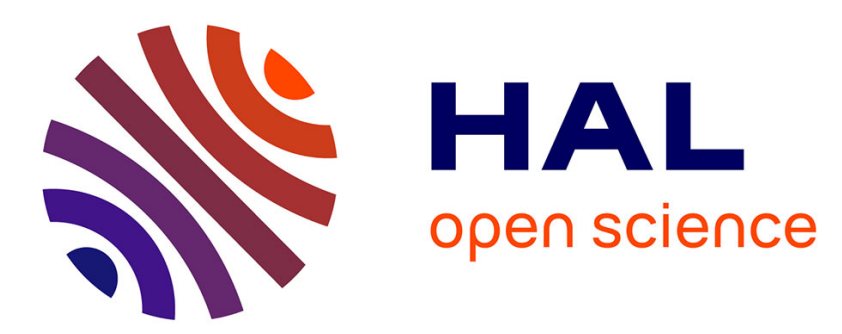

\title{
How the Genome Folds: The Biophysics of Four-Dimensional Chromatin Organization
} Jyotsana J Parmar, Maxime Woringer, Christophe Zimmer

\section{To cite this version:}

Jyotsana J Parmar, Maxime Woringer, Christophe Zimmer. How the Genome Folds: The Biophysics of Four-Dimensional Chromatin Organization. Annual Review of Biophysics and Biomolecular Structure, 2019, Annual Review of Biophysics, 48 (1), 10.1146/annurev-biophys-052118-115638 . pasteur02082840

HAL Id: pasteur-02082840

https://hal-pasteur.archives-ouvertes.fr/pasteur-02082840

Submitted on 5 Mar 2020

HAL is a multi-disciplinary open access archive for the deposit and dissemination of scientific research documents, whether they are published or not. The documents may come from teaching and research institutions in France or abroad, or from public or private research centers.
L'archive ouverte pluridisciplinaire HAL, est destinée au dépôt et à la diffusion de documents scientifiques de niveau recherche, publiés ou non, émanant des établissements d'enseignement et de recherche français ou étrangers, des laboratoires publics ou privés. 


\title{
How the genome folds: biophysics of $4 \mathrm{D}$ chromatin organization
}

\section{Jyotsana J. Parmar, ${ }^{1,2}$ Maxime Woringer, ${ }^{1,2,3,4}$ and Christophe Zimmer ${ }^{1,2}$}

\author{
${ }^{1}$ Unité Imagerie et Modélisation, Institut Pasteur, 75015 Paris, France \\ 2 UMR 3691, CNRS; C3BI, USR 3756, IP CNRS, Paris, France \\ 3 Sorbonne Universités, CNRS, F-75005 Paris, France \\ 4 Department of Molecular and Cell Biology, Li Ka Shing Center for Biomedical \\ and Health Sciences, CIRM Center of Excellence, University of California, \\ Berkeley, CA 94720, USA.
}

Xxxx. Xxx. Xxx. Xxx. YYYY. AA:1-23

https://doi.org/10.1146/((please add article doi))

Copyright (C) YYYY by Annual Reviews. All rights reserved

\section{Keywords}

chromatin, nuclear architecture, Hi-C, imaging, polymer models, transcription.

\section{Abstract}

The genetic information that instructs transcription and other cellular functions is carried by the chromosomes, polymers of DNA in complex with histones and other proteins. These polymers are folded in nuclei five orders of magnitude smaller than their linear length, and many facets of this folding correlate with or are causally related to transcription and other cellular functions. Recent advances in sequencing and imaging based techniques have enabled new views into several layers of chromatin organization. These experimental findings are accompanied by computational modeling efforts based on polymer physics which can provide mechanistic insights and quantitative predictions. Here, we review current knowledge of the main levels of chromatin organization, from the scale of nucleosomes to the entire nucleus, our current understanding of their underlying biophysical and molecular mechanisms, and some of their functional implications. 


\section{Contents}

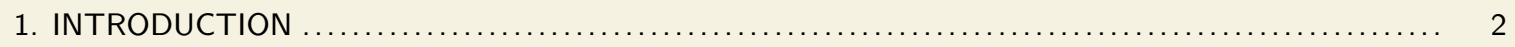

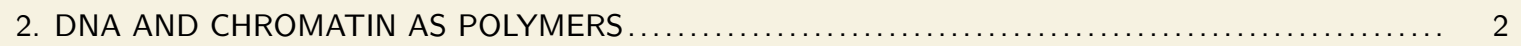

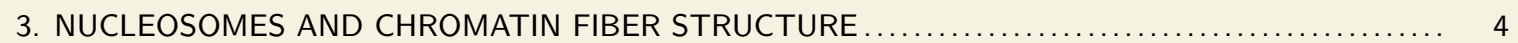

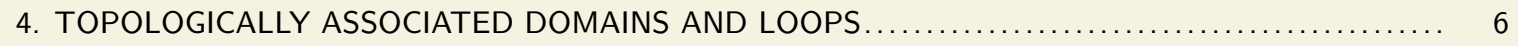

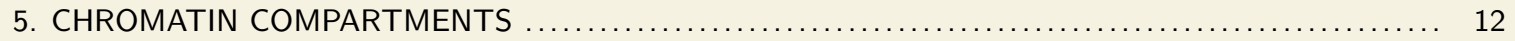

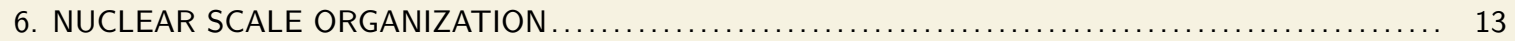

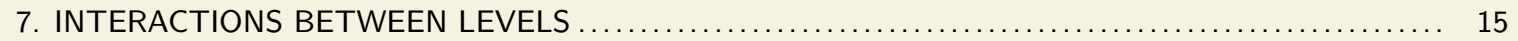

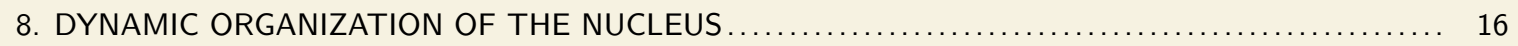

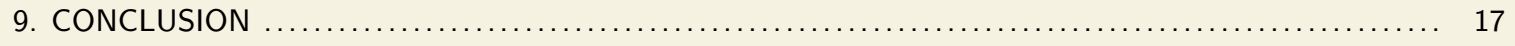

Persistence length: measures the rigidity of a polymer

(Figure 1a); can be defined

geometrically as the length over which tangent vectors remain correlated to each other.

\section{INTRODUCTION}

Eukaryotic genomes, and in particular mammalian genomes are under considerable constraints. The DNA double helix has a diameter of $\sim 2 \mathrm{~nm}$ and, in human cells, would measure $\sim 2 \mathrm{~m}$ long if stretched out, but is folded in a nucleus of $\sim 10 \mu \mathrm{m}$ diameter, thus involving roughly five orders of magnitude of spatial lengths. Despite this tight folding, the genome needs to remain permissible to key biological processes, including DNA replication and gene expression. How genome architecture and biological processes intertwine has puzzled generations of biologists, and a more comprehensive picture of the key determinants of these interactions is just starting to be unravelled. Over the last decade, the field has undergone a dramatic acceleration thanks to the development of powerful sequencing-based assays and microscopy techniques, which have revealed previously unknown levels of chromatin organization. Together with polymer based modeling, these data have helped to uncover some of the fundamental mechanisms that shape chromatin organization. In the following, we first review simple concepts from polymer physics relevant to DNA and chromatin, then discuss four levels of genome organization, from the scale of nucleosomes to the entire nucleus, and highlight proposed biophysical mechanisms and functional consequences.

\section{DNA AND CHROMATIN AS POLYMERS}

The DNA double helix in a typical human chromosome consists of hundreds of millions of base pairs covalently chained together by sugar-phosphate backbones. Thus, DNA perfectly fits the definition of a polymer, as a molecule built from a large number of similar elements (called monomers) bonded together. Polymers obey a wide range of properties that arise solely from the fact that they consist of a large number of monomers and are largely independent of their precise chemical nature. This universality means that many concepts and results from polymer theory $(33,105)$ can be applied to understand the structure and dynamics of DNA and chromatin in cells - we briefly recall some of them next.

A key property of polymers is semi-flexibility: on short length scales, a polymer behaves as a rigid rod, while on larger scales it can bend in arbitrary directions due to thermal agitation alone (Figure 1a). The length scale that separates rigid from flexible behaviour is called 'persistence length'. As a consequence of this semi-flexibility, polymers can adopt an infinite number of $3 \mathrm{D}$ arrangements, or conformations. Individual conformations cannot be predicted, much like the positions of individual molecules in a gas cannot be predicted. How-

Parmar et al. 
(a)
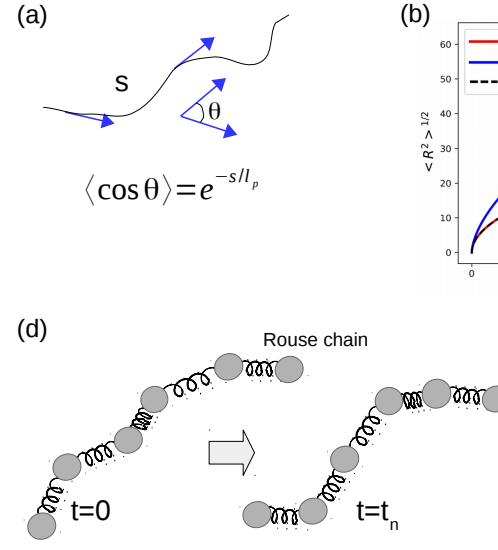

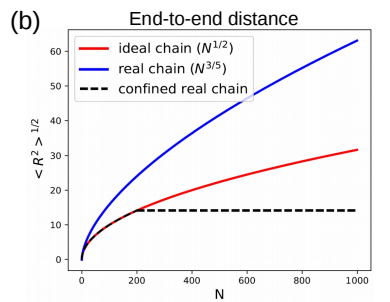

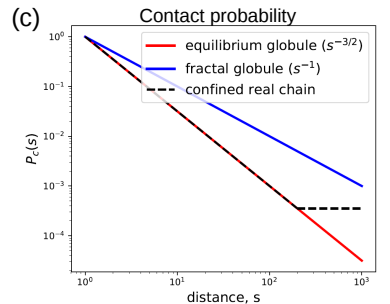

(e) Mean square displacement (MSD)

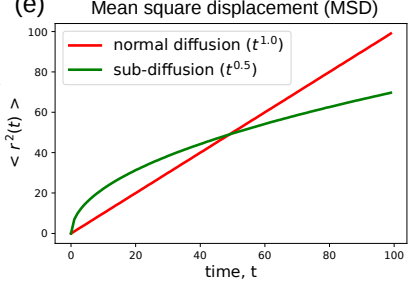

(f) Polymer Model

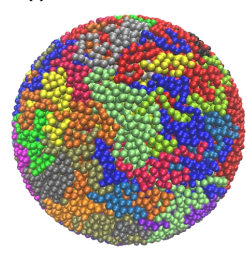

Figure 1

Basic properties of polymers. a) A polymer is a semi-flexible structure whose rigidity can be defined by the bending persistence length $l_{p}$. The average cosine of $\theta$, the angle between the tangent vectors at two loci separated by a curvilinear distance $s$, decreases as $\exp \left(-s / l_{p}\right)$. b) The root mean squared end-to-end distance, $\left\langle R^{2}\right\rangle^{1 / 2}$, as function of the number of monomers $N$, for an ideal chain (red), a real chain (blue), and a chain in confinement (dashed black). c) The contact probability $P_{c}(s)$ as function of curvilinear distance $s$ between loci for an equilibrated polymer (red), a fractal (or crumpled) globule (blue) (53), a confined polymer (dashed black). d) Schematic showing the dynamics of a Rouse polymer where monomers are connected by harmonic springs (105). e) Mean square displacement (MSD) $\left\langle r^{2}(t)\right\rangle$ as function of time for a freely diffusing monomer (red) and a monomer embedded in a polymer chain undergoing Rouse subdiffusion (green). f) Snapshot of a molecular dynamics simulation of multiple chromosomes in the nucleus (J. Parmar, unpublished).

ever, polymer theory can predict statistical quantities, such as the mean distance between the two ends of a polymer chain (Figure 1b) or the frequency with which two monomers contact each other (Figure 1c). These quantities are predicted to obey scaling laws, which describe how they vary with the number of monomers $N$, or equivalently the contour length $s$ of the chain. In the simplest model, the ideal chain, bonds between monomers have random orientations and monomers ignore each other (i.e. neither repel nor attract each other)(105). At equilibrium, in absence of any external constraints or forces, the root mean square end-to-end distance increases as $\sqrt{s}$ (Figure 1b, red), while the contact frequency decays like $s^{-3 / 2}$ (Figure 1c, red). The more realistic 'real chain' model accounts for the fact that a polymer cannot self-intersect. This constraint leads to a swelling of the chain and a faster scaling of end-to-end distances (Figure 1b, blue). In a confined and crowded volume such as the nucleus, however, this swelling can be counterbalanced by the presence of other chains (and other segments of the same chain). In that case, the scaling laws become similar to the ideal chain up to a distance where monomers behave as if they were no longer part of the same chain, at which point they level off (Figure 1b,c, black dashed).

Theory can also predict properties of polymer motions. The simplest model, the Rouse model, describes how the random motion of a single monomer is influenced by that of other monomers to which it is connected (Figure 1d). According to this model, the mean square

Contour length: the distance between two points on a polymer as measured when walking along the chain; can be much larger than the Euclidian distance between these points.

\section{Equilibrium}

(thermodynamic): State of lowest free energy, where there is no net force acting on the system and statistical quantities such as temperature or pressure remain constant. 
Histone

modifications:

Addition of small

molecules (e.g., acetyl, methyl or

phosphate groups)

to different histones

at different amino

acid positions. displacement (MSD) of a monomer grows like the square root of time over short time scales $(105,104)$, unlike a free particle, for which the MSD is simply proportional to time.

These predictions are based on a number of important assumptions, especially equilibrium and a polymer consisting of identical monomers (homopolymer). Neither of these two assumptions holds true for DNA and chromatin fibers, which are subject to ATP-consuming (i.e. energy-driven) processes such as transcription and replication, and have a non-uniform composition determined by the DNA sequence and epigenetic histone modifications. One might therefore expect the above relationships to utterly fail when applied to chromatin fibers in real biological nuclei. Perhaps surprisingly, however, basic homopolymer physics has proven quite effective at explaining some key features of nuclear architecture in a number of organisms (104). Discrepancies between predictions of basic polymer models and observations are useful, because they hint at additional mechanisms of potential biological significance and motivate the development of more realistic and complex models. Examples are heteropolymer simulations, where monomers have distinct types and are subject to different interactions (Figure 1f) defined by DNA sequence or epigenetic information, some of which will be discussed in subsequent sections $(104,46,11,107)$.

\section{NUCLEOSOMES AND CHROMATIN FIBER STRUCTURE}

\section{Beads-on-a-string}

The DNA double helix contains approximately 3 base pairs (bp) per nanometer length and has a persistence length of $\sim 50 \mathrm{~nm}$, i.e. $150 \mathrm{bp}$, as estimated from in vitro experiments (125). According to the ideal chain model (Figure 1b), an average human chromosome would then have an average size exceeding $50 \mu \mathrm{m}$, much larger than typical nuclei. The first level of genome packaging is achieved thanks to nucleosomes, histone octamers whose shape can be approximated by a short cylinder of $10 \mathrm{~nm}$ diameter and $5 \mathrm{~nm}$ height. Despite the stiffness of DNA at this scale, $147 \mathrm{bp}$ of DNA wrap around each nucleosome, taking 1.7 turns. This interaction occurs because the positively charged histones are attracted to the negatively charged DNA, resulting in a net free energy gain of $\sim 40 k_{B} T(71,130)$. Nucleosomes are spaced by 20-40 bp of linker DNA, such that a stretched array of nucleosomes appears as 'beads-on-a-string' in electron micrographs (Figure 3a). Nucleosomes are dynamic and can be repositioned by ATP-dependent chromatin remodelers (132, 79). Several equilibrium and non-equilibrium models, with (un)binding and sliding kinetics, have been successful at explaining the positioning and dynamics of nucleosomal arrays (Figure 3a, blue box $)(63,88,89)$.

\section{The elusive chromatin fiber structure}

How are nucleosomes arranged with respect to each other in 3D? Early in vitro transmission electron microscopy and X-ray experiments suggested that the beads-on-a-string fiber would fold in a higher-order chromatin fiber of $\sim 30 \mathrm{~nm}$ diameter in which nucleosomes are tightly packed together $(41,7,124,51,34,109,102,64,112)$. This structure was thought to arise from the supercoiling of regularly spaced nucleosomes and stabilized by linker histone and electrostatic interactions between the histone tails (102), resulting in DNA compactions far exceeding $100 \mathrm{bp} / \mathrm{nm}$. However, this characteristic structure has not been confirmed in vivo $(99,47,57)$, and more generally, the intermediate folding of chromatin remains elusive. Recent 3D high resolution EM data of specifically labeled chromatin (87) revealed a complex

Parmar et al. 


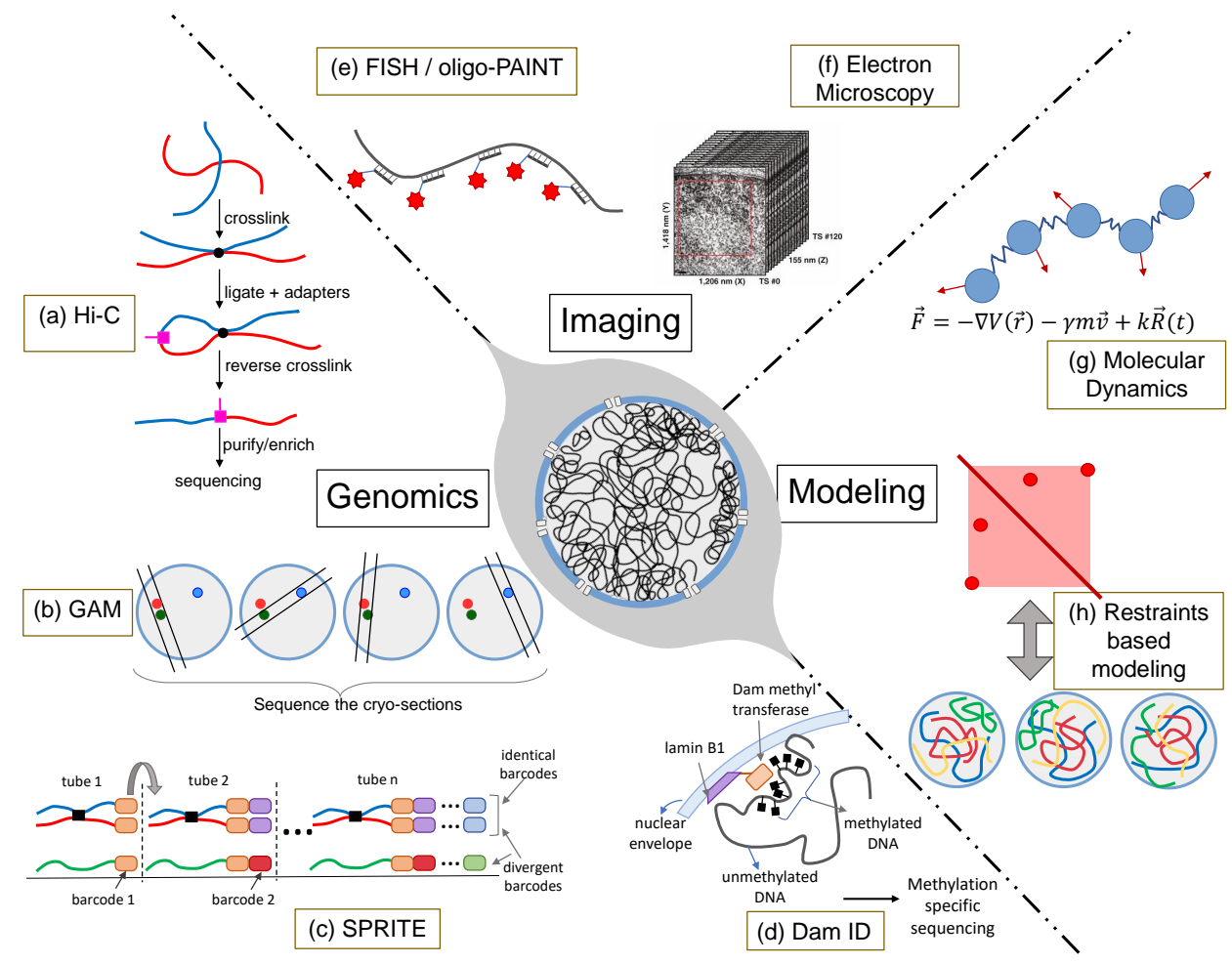

Figure 2

Techniques to study chromatin organization fall in three main categories: genomics, imaging and modeling. Genomics: a) In Hi-C (68), different chromatin regions that are in close spatial proximity are crosslinked, fragmented, ligated and marked with adapters (pink). Fragments are then reverse crosslinked, purified, sequenced and mapped to their genomic locations, yielding genome-wide contact frequency matrices. b) In GAM, nuclei are cryo-sectioned into thin slices and their DNA is sequenced (8). Analysis of locus cooccurrence in many sections allows to infer their proximity, including multi-way interactions, without requiring ligation. c) In SPRITE, DNA and RNA fragments are barcoded in a sequential manner that allows to detect both DNA-DNA and DNA-RNA associations by sequencing (94). d) In Dam-ID, chromatin regions close to the nuclear lamina are marked by the Dam methyl transferase and are mapped genome-wide by sequencing (54). Imaging: e) DNA-FISH methods allow to visualize targeted chromatin domains or entire chromosomes in single cells $(16,9,123)$. f) Electron microscopy, in combination with DNA-specific labeling, can reveal nanometer-scale 3D chromatin structures in frozen samples (87). Modeling: g) Molecular dynamics simulations can model time-dependent changes in chromosome configurations by computing the displacement of monomers based on internal and external forces $(103,104,6)$. Such models can predict contact frequencies and average locus positions from a relatively small number of assumptions. h) Inverse modeling approaches typically use Hi-C data to reconstruct a population of $3 \mathrm{D}$ structures consistent with the data $(61,104)$.

and irregular folding of the DNA, without $30 \mathrm{~nm}$ fiber but with various diameters, ranging from $5 \mathrm{~nm}$ to $24 \mathrm{~nm}$. Recent fluorescence superresolution imaging data are also consistent with the absence of $30 \mathrm{~nm}$ fibers and a polymorphic structure $(101,85)$.

Modeling studies can also provide information about the chromatin fiber structure and estimates of its compaction and rigidity based on contact frequency measurements from 
Hi-C: A genomic technique that maps the contact frequency of any two DNA fragments sufficiently close in space to be captured by crosslinking (Figure 2a).

TAD (Topologically Associated Domain): Chromatin region of $40 k b-3 M b$ size appearing along the diagonal of Hi-C maps as regions of enriched intrachromosomal contacts (Figure 3d, Figure 4a).

Loop: two distant loci of the same chromosome are maintained in close proximity, at least transiently, by other molecules. Identified as peaks in the $\mathrm{Hi}-\mathrm{C}$ map (Figure 3d, Figure 4b).

Stripe/flame: Line of high contact frequency along a TAD border, arising when a locus is in contact with the entire TAD region (Figure 4c).

Cohesin: A ring-like complex previously known for its role in holding the two sister chromatids together after replication (80).
$\mathrm{Hi}-\mathrm{C}$ techniques or its ancestors (30) and distances between loci from imaging. In yeast, an analysis using a whole nucleus simulation inferred an average compaction of $53-65 \mathrm{bp} / \mathrm{nm}$ -also arguing against a $30 \mathrm{~nm}$ fiber- and a persistence length of 52-85nm, i.e. $\sim 3-6 \mathrm{~kb}(6)$. Based on these numbers, the ideal chain model would predict a human chromosomes size of $\sim 15 \mu \mathrm{m}$. This is much larger than the actual size of chromosomes, calling for other explanations, as will be discussed in section 6 .

\section{TOPOLOGICALLY ASSOCIATED DOMAINS AND LOOPS}

\section{TADs, subTADs, loops and stripes}

While electron and light microscopy have allowed insights into the structure of chromatin and chromosomes at small (nucleosome-level) and large (nuclear) scales (see section 6 ), intermediate scales of chromatin folding have long remained obscure. A major milestone enabled by Hi-C was the discovery that intrachromosomal contact frequency matrices display squared blocks of higher frequencies along the diagonal, reflecting regions within which contacts occur more frequently than with any other parts of the genome, and where the average contact frequencies decay slower than the genome-wide average $(32,84)$ (Figure 3b, Figure 4a, Figure 5). These domains were called 'topologically associated domains' (TADs) (although in this context 'topological' does not carry its physical meaning). Although dependent on the exact definition, the number of TADs in human cells has been reported as $\sim 10,000$, with a median size of $\sim 200 \mathrm{~kb}(98)$. While TADs were first characterized in population-averaged Hi-C maps, later imaging experiments support their presence as physical units in single nuclei (114). Another prominent feature uncovered by $\mathrm{Hi}-\mathrm{C}$ are chromatin loops, identified as peaks in the contact maps (Figure $4 \mathbf{b}$ ). A large fraction of TADs have such peaks at their corners and conversely, a large proportion of loops are associated to TADs (98). With sufficient sequencing coverage, smaller TADs can be found nested within bigger ones, and these 'sub-TADs' tend to share common contact peaks. In addition, a large portion of TADs feature "stripes" at their edges, indicating that contacts between a locus at the boundary and all other loci within the TAD are more frequent than between random pairs of loci within the TAD (Figure 4c) (121).

\section{Loop extrusion}

What mechanism can explain the formation of loops, TADs and stripes ? Arguably the simplest scenario is that contacts between distant sites first occur because of random collisions between monomers in a polymer undergoing thermal fluctuations. Most of these random contacts will be short-lived; however if two colliding loci are bound by molecular factors that can form longer lived interactions, these will stabilize contacts and create local peaks in the Hi-C matrix. Indeed, the boundaries of TADs are strongly enriched in specific proteins, most notably the insulator protein CTCF and cohesin (98). However, the random collision model should also generate contact enrichments with other domains on the same chromosome, and even with other chromosomes, leading to off-diagonal blocks in the contact maps - a prediction that is not borne out by Hi-C data. This model also fails to explain another key observation, namely that the boundaries of TADs correlate strongly with converging CTCF sites, i.e. motifs oriented towards each other, while the three other possible orientations of these motifs are strongly disfavored (98). Finally, how TADs with only one stripe can arise in this scenario is similarly unclear. 

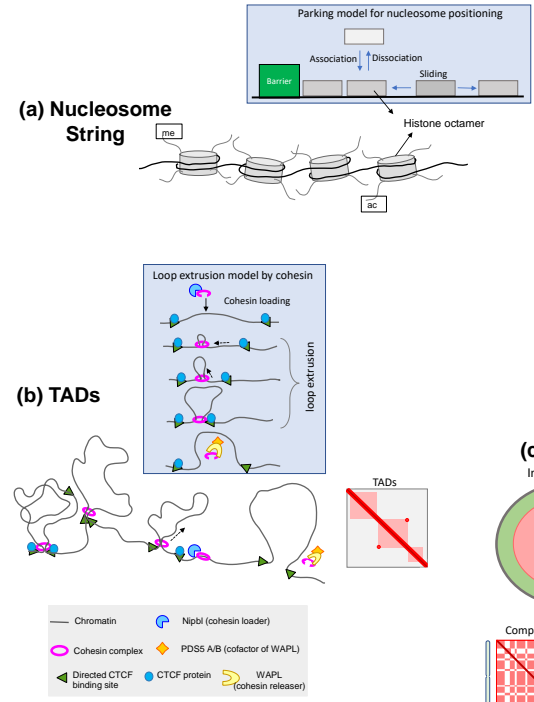
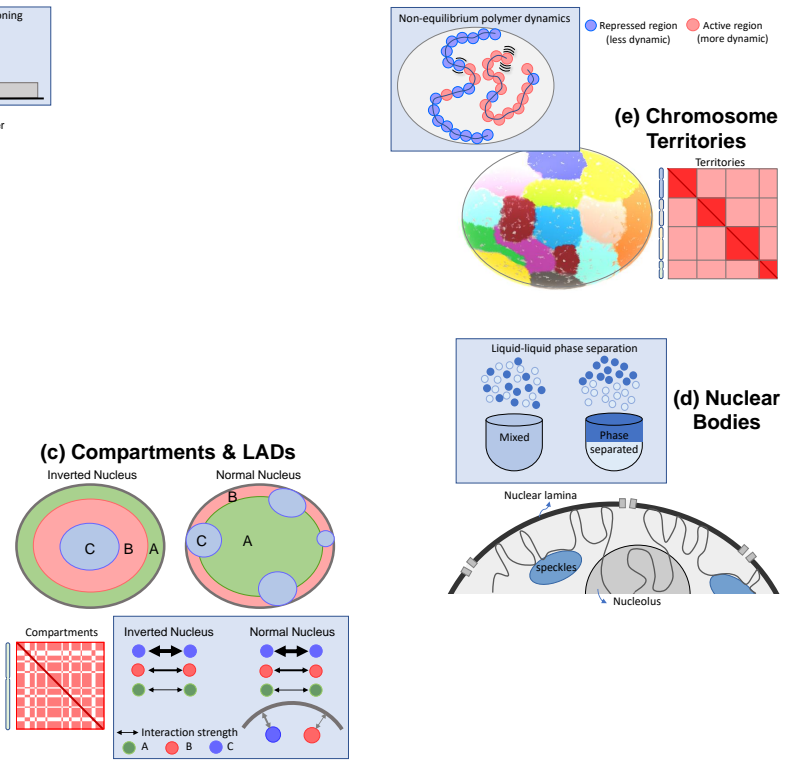

Figure 3

Different levels and mechanisms of chromatin organization. This figure highlights distinct features of 3D genome architecture and some proposed explanations for their formation (boxes).

a) Chromatin fiber: Wrapping of DNA around nucleosomes results in the $\sim 10 \mathrm{~nm}$ beads-on-a-string fiber. The positioning of nucleosomes is determined by energetic barriers (green), steric hindrance by other nucleosomes, and by ATP-dependent chromatin remodelers that can assemble, disassemble or slide nucleosomes (box) $(88,89,24)$. The compaction of nucleosomes in the chromatin fiber is affected by posttranslational modifications of histone tails such as methylation ('me') or acetylation ('ac'). b) TADs and loops: TADs appear as blocks of higher contact frequency on the Hi-C map diagonal. Loops show up as peaks, often located at TAD corners. The box shows chromatin regions undergoing cohesin-mediated loop extrusion (44). In this scenario, cohesin is loaded on DNA by Nipbl, and once loaded extrudes DNA until reaching a properly oriented CTCF boundary. Cohesin can be released from the chromatin by WAPL and PDS5A/B factors. c) Compartments and LADs: euchromatin (A, green), facultative heterochromatin $(\mathrm{B}, \mathrm{red})$ and constitutive heterochromatin $(\mathrm{C}$, blue) compartments segregate radially in the nucleus, as seen by microscopy and the checkerboard pattern of Hi-C maps. LADs strongly correlate with B compartments. In 'inverted nuclei' (111), the radial organization is reversed, but the contact pattern remains similar. Both can be explained by a heteropolymer model that involves attractions between heterochromatic regions, and additional lamina-dependent interactions with the nuclear envelope to model conventional nuclei (box) (38) d) Nuclear bodies, including speckles and nucleoli form membrane-less compartments in the nucleus that appear driven by liquid-liquid phase separation (133). e) Chromosome territories: distinct chromosomes (shown in color) take separate, non-overlapping positions in the nucleus, with the more transcriptionally active chromosomes preferentially occupying the nuclear center (27). Hi-C maps shows high intrachromosomal and low interchromosomal contact frequencies. One proposed mechanism of chromosome territory formation (box) assumes activity-dependent dynamics (red beads: gene rich regions, more dynamic; purple beads: gene poor regions, less dynamic) (48).

By contrast, all of these observations can be simultaneously recapitulated by a very different mechanism known as loop extrusion (80, 4, 98, 106, 45, 86) (Figure 3b and Figure 4). According to this model, cohesin rings land on chromatin and actively pull out (extrude) 
the DNA, until they fall off or encounter obstacles such as CTCF bound sites (with the right orientation) or other cohesin complexes. Molecular dynamics simulations of loop extrusion $(106,45,86,121)$, although assuming some ad-hoc parameters (e.g. an average processivity of $\sim 200 \mathrm{~kb}$, a $10 \%$ permeability of CTCF boundaries and uni-directional or bidirectional movements) are remarkably successful at explaining almost all the experimental evidence mentioned above, as well as the effect of several experimental perturbations summarized next (44).

\section{Molecular and energetic determinants of TADs}

Several lines of evidence directly or indirectly support the loop extrusion model and indicate the role of key molecular determinants of TAD formation. Induced degradation of the cohesin subunit Rad21 leads to complete disappearance of TADs in less than 40 minutes, but restoration of cohesin recovers TADs within 15-40 mins, demonstrating the crucial role of cohesin in TAD formation $(97,128)$ (Figure 5a). Deletion of Nipbl, a protein that loads cohesin on the DNA, results in similar effects (110) (Figure 5c), implying that cohesin must be loaded repeatedly on chromatin to maintain TADs. While $\sim 90 \%$ of cohesin binding sites coincide with CTCF sites, only $29 \%$ coincide with Nipbl sites and only $\sim 11 \%$ of CTCF sites coincide with Nipbl sites indicating that cohesin is loaded outside of CTCF sites and, once loaded, translocates very fast from the loading site to CTCF sites $(134,19,121)$.

Conversely, deletion of WAPL and/or PDS5A and PDS5B, proteins that cooperate to releases cohesin from the DNA, yields an enlargement of TADs by more than $200 \mathrm{~kb}$ and a proliferation of loops (Figure 5d) $(128,55)$. In absence of WAPL, cohesin can travel far distances, even bypass CTCF, resulting in a loss of interphase chromatin organization and characterized by condensed mitotic-like chromatin referred to as "vermicelli" $(115,128$, 19, 55) (Figure 5d). These observations suggest that turnover of cohesin is necessary for proper chromatin organization during interphase, and that if left on chromatin, cohesin keeps extruding longer lengths of DNA resulting in very condensed chromosomes.

The degradation of CTCF protein does not remove TADs but makes their boundaries fuzzier, in accordance with the above idea that CTCF is not directly involved in TAD formation but in defining the boundaries (Figure 5b). Disruption or flipping of CTCF binding sites by genome editing results in changes of TAD boundaries (84), e.g. fusion of consecutive TADs, in excellent agreement with simulation predictions (106). Moreover, disruption of CTCF (in mouse) results in several new cohesin peaks at the active transcription sites (19), suggesting that absence of CTCF allows cohesin to travel longer distances until it finds another roadblock, which in this case could be active transcription. How CTCF or active transcription sites block cohesin is still an open question.

While some models assume that extrusion relies on an energy-driven activity of cohesin as a molecular motor $(45,106)$ others propose extrusion without such activity, powered by either transcriptionally induced supercoiling or even mere thermal diffusion $(95,92)$. Experimentally depleting the cells of ATP shifts the genome-wide distribution of cohesin away from CTCF sites and towards Nipbl binding sites, and prevents the reformation of TADs when restoring cohesin levels after induced degradation. These experiments support an energetic requirement for cohesin translocation from the loading sites and for TAD formation (121).

Further independent evidence in favor of the extrusion model comes from in vitro single molecule imaging experiments showing that the human cohesin complex can translocate on

8 Parmar et al. 
DNA and bypass single nucleosomes and DNA bound proteins, but not CTCF (29). However in this study translocation was independent of ATP, whereas in yeast cohesin loading is ATP-dependent $(76,75,74)$. Another recent single molecule experiment with the related yeast condensin complex showed fast, unidirectional, ATP-dependent loop extrusion ( $\sim 1500 \mathrm{bp} / \mathrm{s}$, step size $\sim 50 \mathrm{~nm})$ on linear DNA $(49)$. The dynamic nature of loops is also supported by single molecule tracking of cohesin and CTCF in vivo (56). Further experimental studies are required to definitively establish active loop extrusion as the mechanism of TAD formation.

Finally, we note that although the role of cohesin in TAD formation is supported by many Hi-C experiments, a recent imaging study showed that even in the absence of cohesin, TAD-like structures remain present in single cells, although their boundaries become randomized along the genome and no longer preferentially associate with CTCF sites (12) . Moreover, the TAD-like domains were reestablished after mitosis in the absence of cohesin. These new results question the role of cohesin in defining chromatin domains in single cells and calls for more investigations.

(a)
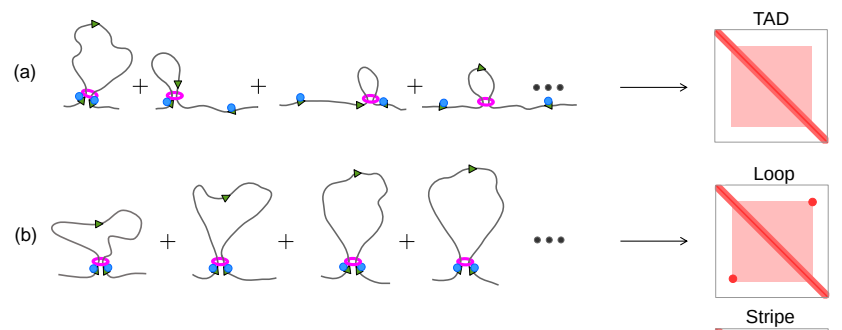

(c)

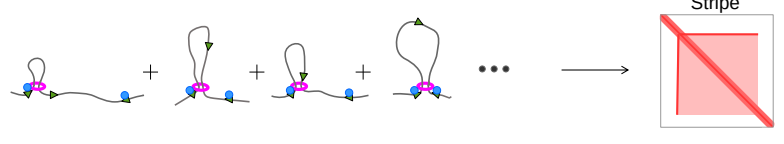

- Chromatin $\quad$ C Cohesin complex $\quad \varangle$ Oriented CTCF site $\quad$ CTCF protein

Figure 4

Loop extrusion scenarii and resulting contact maps. Multiple conformations of the same chromatin region in different cells are shown on the left, the resulting population averaged contact frequency map (i.e. the expected Hi-C map) is shown on the right. a) TAD formation: loops extruded bidirectionally by cohesin landing at random positions generate an enrichment of contacts within a domain (defined by converging CTCF sites) as reflected by a square on the main diagonal of the contact map. b) Loop extrusion stops at converging CTCF sites, giving rise to a contact frequency peak at the TAD corner. c) cohesin landing near a CTCF site and extruding chromatin unidirectionally yields a stripe at the TAD boundary.

\section{Functional role of TADs}

A key property of TADs is their high degree of conservation between cell types and species $(32,98)$. In line with this, TADs and loop extrusion are thought to be both associated and crucial to several biological processes.

Transcription In terms of gene expression, TADs can be seen as highly functional units: genes within the same TAD tend to be coregulated (131) and loops correlate with enhancer- 
Enhancer: A non-coding region of DNA that can exert regulatory control on nearby or distant gene promoters by bringing transcription factors in contact with the transcription pre-initiation machinery

Heterochromatin: initially identified as electron dense chromatin regions by electron microscopy. Subdivided into constitutive heterochromatin (never transcribed) and facultative heterochromatin (reversibly silenced genes).

Euchromatin: initially discovered by electron microscopy as less electron-dense chromatin regions in the nucleus. $\quad 10$ Contains most of the active genes. promoter interactions (98). Alterations of TADs can lead to abnormal expression patterns. For instance, disruption of a single TAD boundary was sufficient to induce polydactyly, a severe development malformation, in mice (70). This effect was explained by the induction of contacts between an enhancer and the promoter of a developmental gene that were previously insulated from each other by the TAD boundary. Similarly, it was shown that loss of CTCF at a TAD boundary in patient-derived cells leads to aberrant contacts of a constitutive enhancer with an oncogene and hyperactivation of its expression, resulting in increased cell proliferation (42). Such evidence underlies the view that TADs provide a means to constrain the action of enhancers to a small number of promoters. However, this immediately raises the question as to how the relatively moderate contact insulation afforded by TADs (contacts within TADs are on average only 2-3 times more frequent than across TADs (73)) can explain the fact that gene expression essentially follows an all-or-nothing behaviour depending on whether both an active enhancer and a promoter reside in the same $\operatorname{TAD}(70,42)$. One possible solution to this conundrum might be the uni-directional extrusion mentioned above to explain contact stripes (121) (Figure 4c). If one of the loop anchors is fixed at a promoter, DNA extrusion can bring this locus in contact sequentially with the entire TAD domain, including all potential enhancer sequences, without having to rely on random 3D collisions. This process might greatly increase the frequency of interactions between pairs of enhancers and promoters sharing a TAD (and a stripe) compared to pairs that do not.

Another potential answer might come from the time scales of promoter activation by enhancer interactions. In the traditional view of mammalian gene expression, a physical contact between the enhancer sequence bound by activating transcription factors and the transcription pre-initiation complex is required to initiate transcription, and many lines of evidence support this model (31). However, it is unclear whether this physical contact is actually required for the RNA polymerase to initiate transcription, or whether it simply potentiates the polymerase, enabling it to initiate transcription later, even in absence of enhancer-promoter contact. In Drosophila, enhancer-promoter contacts and transcription seem to be highly synchronized and more generally, FISH experiments clearly demonstrate a lower enhancer-promoter distance in active genes than in inactive genes, arguing for a direct link between contacts and transcription $(69,21)$. On the other hand, both cohesin and CTCF depletion only show minor effects on gene expression over a 6 hour window, suggesting that on a population scale, transcription is already potentiated and proceeds as before, even in the absence of TAD delimitation $(83,97)$. These findings are corroborated by recent single locus imaging in mouse embryonic stem cells, in which no correlation was found between enhancer-promoter distance and transcript production (3). It also remains unclear to what extent specific histone modifications could mediate this potentiation.

While there is evidence of a role of TADs in regulating transcription, there is also evidence for a reverse role of transcription in TAD organization, such as the fact that a large portion of TAD boundaries is enriched in active RNA polymerase instead of CTCF, and that in Drosophila, TADs are much better defined by active histone marks than by CTCF (119). In general however, the interplay between TADs, loops and transcriptional activation is far from being understood and remains to be further elucidated.

Replication In order to enable the replication of the entire human genome in a few tens of minutes, DNA polymerases initiate replication in parallel at several points along chromosomes, leading to 'replication domains' that grow and merge until the entire chromosome

Parmar et al. 


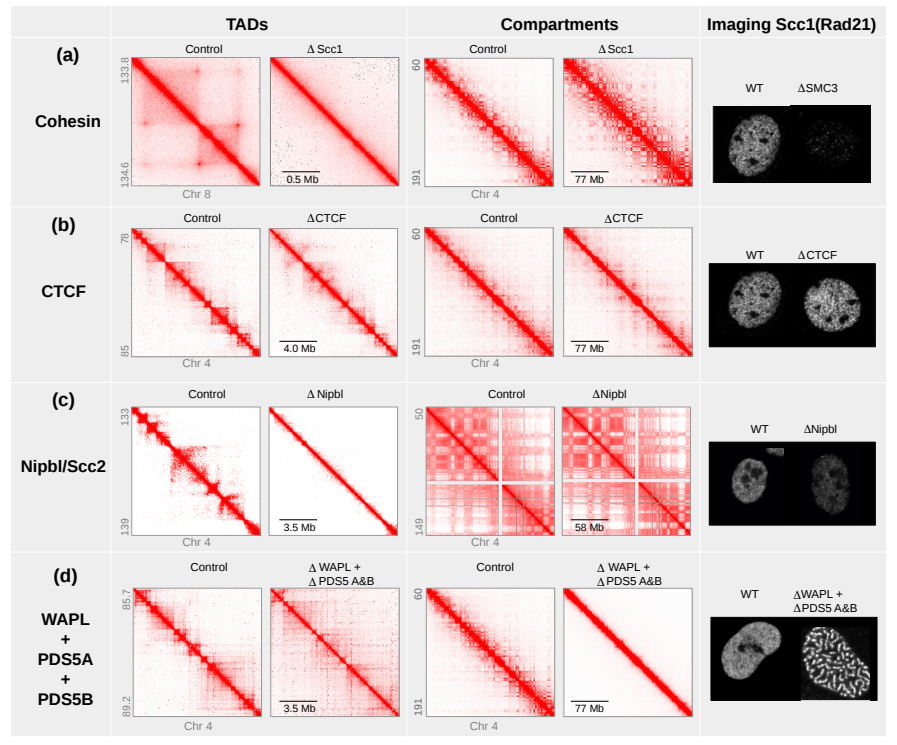

Figure 5

Molecular determinants of chromatin domains. This figure shows the effect of depleting or deleting individual architectural proteins on $\mathrm{Hi}-\mathrm{C}$ contact maps at the scale of TADs and compartments, and on microscopy images of the cohesin component Rad21/Scc1. a) Depletion of Scc1 removes TADs and strengthens compartments. Imaging shows no Scc1 signal when the cohesin subunit SMC3 is deleted, indicating depletion of the cohesin complex. b) CTCF depletion blurs TAD boundaries, but has no effect on compartments. Imaging shows no visible change of Rad21. c) Degradation of the cohesin loader Nipbl has similar effects on TADs and compartments as degradation of cohesin and leads to strongly decreased Rad21 signal . d) Degradation of WAPL along with its cofactors PDS5A and PDS5B results in appearance of new loop peaks, enlargement of TADs, and removal of compartment structures. Imaging of Rad21 shows condensed chromatin structures similar to those seen during mitosis. Depletion of WAPL or the two cofactors alone results in similar, but less pronounced, effects (not shown). Sources of Hi-C data: a: HCT116 (human) (97); b,d: HeLa (human) (128); c: Hepatocytes (mouse) (110).

Visualization of contact maps was done with Juicebox (36). Source of microscopy images: a,b: Embryonic fibroblasts (mouse) (19); c: HAP1 cells (human) (55); d: HeLa (human) (128).

is replicated. Surprisingly, TADs coincide almost perfectly with replication domains (93), suggesting that TADs might also orchestrate replication, in addition to transcription. This colocalization of TADs and replication origins has recently been used in order to visualize TAD dynamics in live cells (129). One recent study found that the progressive establishment of TADs in early zygote development was prevented by replication inhibition as opposed to transcription inhibition (62), but another found that loop domains can reform after restoration of cohesin despite inhibition of replication (121). Clearly, more work is needed to address the mechanistic links between replication and TAD formation. 
Compartments: Megabase-scale regions of the genome, originally evidenced by checkerboard patterns in $\mathrm{Hi}-\mathrm{C}$ maps, representing domains of increased interactions (Figure 3c).

ChIP-seq: An immunoprecipitation technique that provides the genomic locations of where a protein of interest binds.

Lamina-associated domains (LADs): Megabase-size chromatin domains that were shown to physically interact with the nuclear lamina by Dam-ID (Figure 2d).

\section{CHROMATIN COMPARTMENTS}

\section{$A$ and $B$ compartments and LADs}

Electron microscopy images of interphase nuclei typically show dense heterochromatic regions near the nuclear envelope and around nucleoli, with less compact, euchromatin in the center of the nucleus and beneath nuclear pores. A notable exception to this general rule are retinal cells of nocturnal mammals, which display an 'inverted nuclear architecture' where euchromatin relocates to the periphery while constitutive heterochromatin resides at the nuclear center (111). New views into this partitioning of the genome were opened by the identification of chromatin 'compartments' in the first Hi-C study (68). Compartments appear as a checkerboard pattern in the Hi-C map after correcting for the average dependence of contact frequencies on genomic distance (the pattern becomes more apparent when computing the corresponding correlation matrix) (68) (Figure 3c, Figure 5). This pattern, and an eigenvector analysis, suggest that the entire genome is partitioned to first order in alternating regions of a few megabases belonging to two compartments (called A and B). (Subsequent Hi-C experiments with higher resolution further refined this partitioning into six sub-compartments (98)). Unlike for TADs, a locus belonging to the A compartment exhibits enriched contacts with other loci from the A compartment throughout the genome, but has less frequent contacts with loci from the B compartment (and vice-versa). Comparisons with ChIP-seq data shows a strong correlation of A regions with transcriptionally active histone marks and decondensed chromatin, while B regions correlate with inactive histone marks and dense regions, thereby providing a new definition of euchromatin and heterochromatin. Imaging experiment have later confirmed the existence of $\mathrm{A}$ and $\mathrm{B}$ compartments (as well as TADs) with different levels of compaction in single cells of Drosophila (123). The B compartment identified by Hi-C also exhibits very high correlation with 'lamina associated domains' (LADs), chromatin regions in contact with the nuclear envelope, as identified by the Dam-ID technique $(54,120)$ (Figure 2d).

\section{Compartmentalization mechanisms}

Although A and B compartments were identified several years before TADs, our understanding of the mechanisms underlying their segregation is comparatively less advanced. Most explanatory models derive from the observation that A and B compartments are enriched for specific histone modifications (68). These modified histones can act as scaffolds for other proteins capable of interacting with more than one histone, or proteins exhibiting a high level of self-interaction, both of which can potentially mediate phase-separation (37). Alternatively, it has been proposed that compartment segregation might result from associations to (at least) two types of anchors: the nuclear lamina, which contains several proteins known to interact with modified histones and nuclear speckles, which are located more centrally (see section 6$)(22,23,120)$. Other proposed models invoke differences in chromatin dynamics or transcriptional activity (48).

In recent studies $(86,38,92)$, chromosomes were modeled as heteropolymers (Figure 3c) partitioned into three compartments based on the Hi-C maps ( $\mathrm{A}, \mathrm{B}$, plus constitutive heterochromatin, termed $\mathrm{C}$ ). The simulations were able to recover the inverted nuclear architecture mentioned above as well as the compartment organization by assigning realistic interactions between the three compartments, e.g. an attractive energy potential of $0.5 k_{B} T$ for interactions between B-compartment monomers of $30 \mathrm{~kb}$. Keeping the same model, but adding interactions of $\mathrm{B}$ and $\mathrm{C}$ monomers with the nuclear envelope allowed to recover the 
conventional nuclear architecture. This suggests that the compartment segregation may be explained by attractive interactions between heterochromatin regions rather than between euchromatin regions and is unrelated to tethering at the nuclear lamina, and that the default organization of chromatin (in absence of tethering with the lamina) is the inverted one (38). Although the model does not specify the molecular nature of the assumed heterochromatic interaction, one plausible candidate is heterochromatin protein 1- $\alpha$ (HP1 $\alpha$ ), which has been shown to undergo phase-separation, both in vitro (66) and in vivo (113). Clearly, more work is needed to expand on such early results and fully address the molecular and physical mechanisms that underly chromatin compartmentalization at the megabase scale.

\section{Functional implications}

The partitioning of the genome in compartments and LADs correlates with functional processes: B compartments and LADs tend to be transcriptionally inactive and late replicating, A compartments tend to transcriptionally active and early replicating. A central question is to what extent these correlations reflect cause or consequences. Segregation provides spatially separated subcompartments that are amenable to different types of reactions, with different kinetics (127). For example, the high density of heterochromatin, as evidenced e.g. by recent electron microscopy data (87), might prevent the assembly of the multi-Megadalton preinitiation complex and thereby silence gene expression. Consistent with this view, experimental tethering of genes to the nuclear membrane can lead to their transcriptional repression $(100,1)$. On the other hand, experimentally induced chromatin decondensation by recruitment of an acidic peptide did not lead to transcriptional activation, arguing against a direct link between chromatin compaction and gene expression (118). Thus, a complete picture is missing and future research should further explore the causal relations between compartments, LADs and gene expression.

\section{NUCLEAR SCALE ORGANIZATION}

\section{Chromosome territories}

After mitosis, chromosomes decondense and owing to their large size, limited compaction and flexibility would be expected to intermingle and fill out the entire nucleus (104). However, microscopy has shown that the interphase nuclei of many mammalian cells are partitioned into largely disjoint 'chromosome territories' (27) (Figure 3e), whose radial positions display statistical preferences: for instance, gene rich chromosomes tend to occupy central positions while gene poor chromosomes are more peripheral $(28,16)$. In yeast, by contrast, chromosomes strongly intermingle despite their much smaller size $(10,117)$.

Mechanisms: What might explain the different organization of chromosomes in these organisms? One line of explanation is based on the time scale needed for chromosome to relax after mitosis. Because topological constraints (the assumed inability of distinct polymer chains to cross each other) increase the relaxation time of polymers as the third power of their length, this time was predicted to be much longer than the cell cycle for mammalian genomes, but not for yeast. Thus, human chromosomes might simply not have enough time to equilibrate and mix and interphase territories could reflect the individuality of mitotic chromosomes (103). Support for the prediction that mammalian chromosomes are in an out-of-equilibrium state came from the first Hi-C study, which indicated that the genome-
Chromosome territories: first identified as largely non-overlapping nuclear substructures by FISH experiments using probes that specifically cover entire chromosomes 
Nucleolus: The largest, membrane-less organelle of the nucleus, contains a low amount of DNA and a high level of ribosomal RNA.

Nuclear speckles: Small membrane-less compartments positioned close to the nuclear center and involved in mRNA splicing

Liquid-liquid phase separation: a thermodynamic process where two immiscible liquids progressively segregate into two phases, like oil and water (also termed demixing or coacervation) wide averaged contact frequency is inversely proportional to genomic distance $s$ (that is, $P_{c}(s) \propto s^{-1}$ ) (at least between $\sim 500 \mathrm{~kb}$ and $\sim 5 \mathrm{Mb}$ ) in contrast to the $P_{c}(s) \propto s^{-3 / 2}$ scaling expected for equilibrium (Figure 1c) (68). A fractal (or crumpled) globule model, where the polymer remains unentangled in contrast to an equilibrated model was proposed to explain this scaling (53). Conversely, the prediction that yeast chromosomes are at equilibrium is supported by the fact that a Brownian dynamics simulation can successfully account for imaging and Hi-C data, including the contact frequency scaling $(126,6)$.

However, despite the attractiveness of these generic and largely parameter-free models, several questions remain. First, the action of topoisomerase II -which cuts both strands of the DNA double helix- was ignored in these simulations, but is expected to strongly reduce equilibration time by relaxing topological constraints. Whether chromosome territory formation can be explained with realistic modeling of topoisomerase II action remains to be seen, particularly for post-mitotic cells such as neurons. Second, the incomplete relaxation model (103) assumed homopolymers and both ignored the presence of compartments, TADs and differences in transcriptional activity, which other models predict to affect chromosome positioning (48). It will therefore be interesting to revisit the formation of chromosome territories in the context of interactions (e.g. electrostatic (113)) between compartments, loop extrusion, and differential dynamics, all of which will affect the size of interphase chromosomes, even at steady-state.

Functional implications Much as for A/B chromatin compartments, the functional relevance of chromosome territories remains unclear. It has been proposed that chromosome territories facilitate chromosome condensation prior to mitosis (103). Moreover, because the spatial proximity of loci or chromosomes correlates with increased translocations, a hallmark of cancer cells, it has been proposed that the organization in territories acts to minimize inter-chromosomal rearrangements $(82,17)$.

\section{Nuclear bodies}

Nuclear bodies are subcompartments of the nucleus that lack a bona fide membrane. They are usually visible by phase contrast microscopy as spheroid, often dynamic structures, consisting of a dense aggregate of proteins, RNA and potentially many other macromolecules and are associated to specific functions, such as transcription of certain genes, splicing, DNA damage repair etc. (Figure 3d). The prototypical nuclear body is the nucleolus, the site of ribosomal RNA biogenesis (15). Since its discovery, many other ubiquitous or species-specific nuclear bodies have been identified, including nuclear speckles, Cajal bodies, PML bodies, histone locus bodies, and paraspeckles (72). The nucleation and/or morphology of nuclear bodies intimately depends on their activity, for example inhibition of rDNA transcription strongly reduces the nucleolar volume (117), and can be regulated by posttranslational modifications such as phosphorylation of RNA polymerase II (65).

Mechanisms The mechanisms underlying nuclear body formation are under active investigation, with increased attention being focused on liquid-liquid phase separation (59) (Figure 3d). Several examples of phase separation inside the nucleus have been reported, often mediated either by proteins bearing unstructured domains (113, 66), RNAs (39), or other small molecules $(5,90)$. For example, the C-terminal domain of RNA polymerase II has been shown in vitro to perform reversible and regulatable phase-separation (65) and recent

14 Parmar et al. 
evidence suggests that similar mechanisms exist in vivo as well $(25,13)$. Another example, as mentioned above, is HP1 $\alpha$, which was observed to form droplets in live cell fluorescence microscopy of Drosophila (113). In addition, dephosphorylation promotes droplet disassembly, suggesting a mechanism by which demixing can be regulated. A challenge for the coming years is to integrate quantitative models of phase separation with the additional constraints imposed by the physics of polymers (104).

Functions Although lacking a strict membrane, nuclear bodies can sequester some molecules and exclude others, thereby acting as chemical reactors to catalyze specific reactions, decoupling them from different pathways. An interesting property of nuclear bodies created by phase separation is that their very existence can depend on whether its molecular constituents exceed a concentration threshold. This non-linear behaviour could potentially endow the cell with a 'switch'-like response to external stimuli (133).

Another potential function, or consequence, of nuclear body formation is the very organization of chromatin itself. Indeed, it has been shown that chromatin organizes nonrandomly around nuclear bodies. While it is difficult to chart sequences in close proximity of a nuclear body with traditional methods, novel techniques have recently been developed that dispense of proximity ligation to probe for DNA sequences located within much larger distances of other DNA or RNA sequences ((94), Figure 2c,d) or even a specific protein $(22,23)$. These techniques reveal a key role of nuclear speckles, and either LADs $(22,23)$ or nucleoli (94) in overall chromatin organization, and suggest a plausible mechanism for locus positioning that merits further investigation.

\section{INTERACTIONS BETWEEN LEVELS}

A natural question is how the different levels of organization discussed above interact, either cooperatively or antagonistically, with each other. Since chromosomes are single connected structures, one might surmise that these levels are all closely interleaved and hard to disentangle. Therefore, studies able to peel off individual layers of organization are particularly instructive. One striking recent example is the experimentally demonstrated partial decoupling of TADs and compartments (110, 97). In absence of cohesin or its loader Nipbl, the segregation of A and B compartments not only subsists, but is actually strengthened (Figure 5a,c): boundaries between compartments become sharper, and their correlation with histone modifications increases. Conversely, deleting the cohesin release factors (WAPL, and/or PDS5A/B) leads to an enlargement of TADs and a destruction of A/B compartments (Figure 5d) (128). These experiments indicate that the formation of TADs and compartments rely on distinct mechanisms, that partly counteract each other. A similar antagonism is at work between the mechanism that tend to keep chromosomes in distinct territories and those that create $\mathrm{A} / \mathrm{B}$ compartments, which tend to mix regions of different chromosomes belonging to the same compartment. Another salient finding is the above mentioned study showing that A/B compartments subsist in absence of tethering to the nuclear lamina, hence decoupling compartmentalization from LADs (38).

Although a general model able to link all four levels of chromatin organization, from nucleosomes to the entire nucleus, is still lacking, such experiments and modeling approaches point to future unifying frameworks. Further studies are needed to disentangle the competing forces that shape chromatin architecture in the context of transcription and other functional processes. 


\section{DYNAMIC ORGANIZATION OF THE NUCLEUS}

The 3D architecture of the genome is by no means static, since chromatin is in constant motion, as expected from basic polymer dynamics (Figure 1e) and evidenced by live cell microscopy in yeast $(91,58)$ and mammalian cells $(26,20)$. By itself, this mobility leads to stochastic variations in chromosome configurations in cell populations. The variability in chromatin organization can also be analyzed in fixed cells using imaging (14) or single cell HiC methods $(77,96)$, or even inferred from population Hi-C by computational reconstruction methods (61) (Figure 2h). In addition to the dynamics over short time scales in interphase cells, chromatin organization changes dramatically during each cell division, and is altered during differentiation, as briefly discussed next.

\section{Chromatin organization during the cell cycle}

It has long been known from light microscopy that chromosomes undergo major structural changes during mitosis, when the sister chromatids condense, align on a metaphase plate, before being pulled apart into the two future daughter cells. New insights into how chromosome structure changes during the cell cycle have been obtained by $\mathrm{Hi}-\mathrm{C}$ in mammalian and yeast genomes $(81,52,78,60,67,108)$. A first analysis of synchronised cells showed that both TADs and compartments remain similar throughout interphase, with only moderate changes in strength, but little changes in boundaries, yet are completely lost during mitosis. Mitotic chromosomes assume a universal folding structure independent of cell type that was well described by an array of random, $\sim 100 \mathrm{~kb}$ long loops (81). In a subsequent study (52), the timeline was further refined to analyze the successive stages of mitosis every few minutes and the role of condensin proteins in shaping mitotic chromosomes. Especially noteworthy was the appearance of a second diagonal in the Hi-C matrix at pro-metaphase, indicative of a helical chromosome structure, which disappeared upon degradation of condensin II. To explain their Hi-C and imaging data, the authors developed sophisticated polymer models where condensin II creates progressively bigger loops of up to $\sim 700 \mathrm{~kb}$ by the same extrusion process previously discussed for cohesin (section 4) and in agreement with single molecule experiments showing condensin mediated extrusion (49). These big loops are further folded into smaller $(\sim 80 \mathrm{~kb})$ loops extruded by condensin I. The condensin II loop anchors are assumed to form a scaffold that adopts a helical structure from which the nested loops emanate radially in a 'spiral staircase' arrangement. Interestingly, a super-resolution imaging study of condensins I and II in mitotic chromosomes provides independent support for the nested loop arrangement (although the helicity could not be ascertained) with similar quantitative estimates of loop sizes (122). Importantly, even though deletion of condensin I and II results in some morphological changes in mitotic chromosomes, chromosomes remain condensed suggesting the involvement of some other unknown proteins in mitotic condensation $(52)$.

\section{Chromatin reorganization during early development}

Although interphase chromatin organization is completely lost during mitosis, it is reestablished in the daughter nuclei. The restoration of TADs can be explained by cohesin loading and loop extrusion, since the CTCF binding sites that demarcate most TAD boundaries are encoded in the DNA. Similarly, the reestablishment of compartments can be determined by histone marks which are inherited after mitosis $(2,116,40)$. However the question remains 
how chromatin organization is established in the first place during the early stage of development. Recent single cell (or low input) Hi-C studies in mouse oocytes and zygotes after fertilization provide some initial views into this process $(43,50,35)$. Interestingly, Hi-C data show a marked compartmentalization in mouse sperm that lacks in oocytes. This striking difference is attributed to (i) differences in compaction (tightly compacted paternal genome vs more decondensed maternal genome) and (ii) differences in transcription during G1 phase. However after fertilization the difference is gradually decreased and after 8 cell divisions both maternal and paternal genomes acquire full fledged A/B compartments. Future work may use single cell transcriptomics approaches to further understand the links between the observed chromatin reorganizations and transcription in early developmental stages.

\section{FUTURE ISSUES}

1. Model the polymorphic chromatin fiber from the scale of nucleosomes to entire chromosomes.

2. Visualize loop extrusion in live cells. Understand how TADs affect enhancerpromoter interactions and initiate or potentiate transcription.

3. Determine the molecular mechanisms that promote the segregation of $\mathrm{A} / \mathrm{B}$ compartments.

4. Understand the formation of chromosome territories and nuclear bodies in the context of compartments, TADs and phase separation.

\section{CONCLUSION}

In this review, we have tried to summarize current knowledge about the $4 \mathrm{D}$ organization of the chromatin fiber, our understanding of its mechanisms and some of the functional consequences of this organization, while also pointing to open questions and future research. Although necessarily incomplete, we hope that this review will help stimulate further work in this highly dynamic field at the crossroads of genetics, cell biology and physics.

\section{DISCLOSURE STATEMENT}

The authors are not aware of any affiliations, memberships, funding, or financial holdings that might be perceived as affecting the objectivity of this review.

\section{ACKNOWLEDGMENTS}

We thank Elena Rensen for useful comments on the manuscript. This work was funded by Institut Pasteur and Fondation pour la Recherche Médicale en France (Equipe FRM, DEQ 20150331762).

\section{LITERATURE CITED}

1. Akhtar W, de Jong J, Pindyurin AV, Pagie L, Meuleman W, et al. 2013. Chromatin position effects assayed by thousands of reporters integrated in parallel. Cell 154:914-927 
2. Alabert C, Barth TK, Reverón-Gómez N, Sidoli S, Schmidt A, et al. 2015. Two distinct modes for propagation of histone PTMs across the cell cycle. Genes and Development 29:585-590

3. Alexander JM, Guan J, Huang B, Lomvardas S, Weiner OD. 2018. Live-Cell Imaging Reveals Enhancer-dependent Sox2 Transcription in the Absence of Enhancer Proximity. bioRxiv :1-42

4. Alipour E, Marko JF. 2012. Self-organization of domain structures by DNA-loop-extruding enzymes. Nucleic Acids Research 40:11202-11212

5. Altmeyer M, Neelsen KJ, Teloni F, Pozdnyakova I, Pellegrino S, et al. 2015. Liquid demixing of intrinsically disordered proteins is seeded by poly(ADP-ribose). Nature Communications 6

6. Arbona JM, Herbert S, Fabre E, Zimmer C. 2017. Inferring the physical properties of yeast chromatin through Bayesian analysis of whole nucleus simulations. Genome Biology 18:1-15

7. Bak AL, Zeuthen J, Crick FH. 1977. Higher-order structure of human mitotic chromosomes. Proceedings of the National Academy of Sciences 74:1595-9

8. Beagrie RA, Scialdone A, Schueler M, Kraemer DC, Chotalia M, et al. 2017. Complex multienhancer contacts captured by genome architecture mapping. Nature 543:519-524

9. Beliveau BJ, Boettiger AN, Avendaño MS, Jungmann R, McCole RB, et al. 2015. Singlemolecule super-resolution imaging of chromosomes and in situ haplotype visualization using Oligopaint FISH probes. Nature Communications 6:1-13

10. Berger AB, Cabal GG, Fabre E, Duong T, Buc H, et al. 2008. High-resolution statistical mapping reveals gene territories in live yeast. Nature Methods 5:1031-1037

11. Bianco S, Chiariello AM, Annunziatella C, Esposito A, Nicodemi M. 2017. Predicting chromatin architecture from models of polymer physics. Chromosome Research 25:25-34

12. Bintu B, Mateo LJ, Su JH, Sinnott-Armstrong NA, Parker M, et al. 2018. Super-resolution chromatin tracing reveals domains and cooperative interactions in single cells. Science 362

13. Boehning M, Dugast-Darzacq C, Rankovic M, Hansen AS, Yu T, et al. 2018. RNA polymerase II clustering through carboxy-terminal domain phase separation. Nature Structural and Molecular Biology 25:833-840

14. Boettiger AN, Bintu B, Moffitt JR, Wang S, Beliveau BJ, et al. 2016. Super-resolution imaging reveals distinct chromatin folding for different epigenetic states. Nature 529:418-422

15. Boisvert FM, van Koningsbruggen S, Navascués J, Lamond AI. 2007. The multifunctional nucleolus. Nature reviews Molecular cell biology 8:574

16. Bolzer A, Kreth G, Solovei I, Koehler D, Saracoglu K, et al. 2005. Three-dimensional maps of all chromosomes in human male fibroblast nuclei and prometaphase rosettes. PLoS Biology 3:0826-0842

17. Branco MR, Pombo A. 2006. Intermingling of chromosome territories in interphase suggests role in translocations and transcription-dependent associations. PLoS Biology 4:780-788

18. Broedersz CP, Mackintosh FC. 2014. Modeling semiflexible polymer networks. Reviews of Modern Physics 86:995-1036

19. Busslinger GA, Stocsits RR, Van Der Lelij P, Axelsson E, Tedeschi A, et al. 2017. Cohesin is positioned in mammalian genomes by transcription, CTCF and Wapl. Nature 544:503-507

20. Chen B, Gilbert LA, Cimini BA, Schnitzbauer J, Zhang W, et al. 2013. Dynamic imaging of genomic loci in living human cells by an optimized crispr/cas system. Cell 155:1479-1491

21. Chen H, Levo M, Barinov L, Fujioka M, Jaynes JB, Gregor T. 2018. Dynamic interplay between enhancerpromoter topology and gene activity. Nature Genetics 50:1296-1303

22. Chen W, Yan Z, Li S, Huang N, Huang X, et al. 2018. RNAs as Proximity-Labeling Media for Identifying Nuclear Speckle Positions Relative to the Genome. iScience 4:204-215

23. Chen Y, Zhang Y, Wang Y, Zhang L, Brinkman EK, et al. 2018. Mapping 3D genome organization relative to nuclear compartments using TSA-Seq as a cytological ruler. Journal of Cell Biology 217:4025-4048

24. Chereji RV, Clark DJ. 2018. Major determinants of nucleosome positioning. Biophysical journal

25. Chong S, Dugast-darzacq C, Liu Z, Dong P, Dailey GM, et al. 2018. Imaging dynamic and 
selective low-complexity domain interactions that control gene transcription. Science 2555:117

26. Chubb JR, Boyle S, Perry P, Bickmore WA. 2002. Chromatin motion is constrained by association with nuclear compartments in human cells. Current Biology 12:439-445

27. Cremer T, Cremer M. 2010. Chromosome territories. Cold Spring Harbor perspectives in biology $2: 1-22$

28. Croft JA, Bridger JM, Shelagh B, Perry P, Teague P, Bickmore WA. 1999. Differences in the localization and morphology of chromosomes in the human nucleus. Journal of Cell Biology 145:1119-31

29. Davidson IF, Goetz D, Zaczek MP, Molodtsov MI, Huis in 't Veld PJ, et al. 2016. Rapid movement and transcriptional relocalization of human cohesin on DNA. The EMBO Journal 35:2671-2685

30. Dekker J, Rippe K, Dekker M, Kleckner N. 2002. Capturing chromosome conformation. Science 295:1306-1311

31. Deng W, Rupon JW, Krivega I, Breda L, Motta I, et al. 2014. Reactivation of developmentally silenced globin genes by forced chromatin looping. Cell 158:849-860

32. Dixon JR, Selvaraj S, Yue F, Kim A, Li Y, et al. 2012. Topological domains in mammalian genomes identified by analysis of chromatin interactions. Nature 485:376-380

33. Doi M, Edwards S. 1988. The Theory of Polymer Dynamics. International series of monographs on physics. Clarendon Press

34. Dorigo B, Schalch T, Kulangara A, Duda S, Schroeder RR, Richmond TJ. 2004. Nucleosome arrays reveal the two-start organization of the chromatin fiber. Science 306:1571-1573

35. Du Z, Zheng H, Huang B, Ma R, Wu J, et al. 2017. Allelic reprogramming of 3D chromatin architecture during early mammalian development. Nature 547:232-235

36. Durand NC, Robinson JT, Shamim MS, Machol I, Mesirov JP, et al. 2016. Juicebox provides a visualization system for hi-c contact maps with unlimited zoom. Cell systems 3:99-101

37. Erdel F, Rippe K. 2018. Formation of Chromatin Subcompartments by Phase Separation. Biophysical Journal 114:2262-2270

38. Falk M, Feodorova Y, Naumova N, Imakaev M, R. BL, et al. 2018. Heterochromatin drives organization of conventional and inverted nuclei Martin. bioRxiv :1-19

39. Feric M, Vaidya N, Harmon TS, Mitrea DM, Zhu L, et al. 2016. Coexisting Liquid Phases Underlie Nucleolar Subcompartments. Cell 165:1686-1697

40. Festuccia N, Dubois A, Vandormael-Pournin S, Tejeda EG, Mouren A, et al. 2016. Mitotic binding of esrrb marks key regulatory regions of the pluripotency network. Nature cell biology 18:1139

41. Finch JT, Klug A. 1976. Solenoidal model for superstructure in chromatin. Proceedings of the National Academy of Sciences 73:1897-1901

42. Flavahan WA, Drier Y, Liau BB, Gillespie SM, Venteicher AS, et al. 2016. Insulator dysfunction and oncogene activation in IDH mutant gliomas. Nature 529:110-114

43. Flyamer IM, Gassler J, Imakaev M, Ulyanov SV, Abdennur N, et al. 2017. Single-nucleus Hi-C reveals unique chromatin reorganization at oocyte-to-zygote transition. Nature 544:110-114

44. Fudenberg G, Abdennur N, Imakaev M, Goloborodko A, Mirny L. 2018. Emerging Evidence of Chromosome Folding by Loop Extrusion. Cold Spring Harbor Symposia on Quantitative Biology LXXXII:1-11

45. Fudenberg G, Imakaev M, Lu C, Goloborodko A, Abdennur N, Mirny LA. 2016. Formation of Chromosomal Domains by Loop Extrusion. Cell Reports 15:2038-2049

46. Fudenberg G, Mirny LA. 2012. Higher-order chromatin structure: bridging physics and biology. Current opinion in genetics 85 development 22:115-124

47. Fussner E, Djuric U, Strauss M, Hotta A, Perez-Iratxeta C, et al. 2011. Constitutive heterochromatin reorganization during somatic cell reprogramming. The EMBO journal 30:177889 
48. Ganai N, Sengupta S, Menon GI. 2014. Chromosome positioning from activity-based segregation. Nucleic Acids Research 42:4145-4159

49. Ganji M, Shaltiel IA, Bisht S, Kim E, Kalichava A, et al. 2018. Real-time imaging of DNA loop extrusion by condensin. Science 7831:1-9

50. Gassler J, Brandão HB, Imakaev M, Flyamer IM, Ladstätter S, et al. 2017. A mechanism of cohesindependent loop extrusion organizes zygotic genome architecture. The EMBO Journal 36:e201798083

51. Gerchman SE, Ramakrishnan V. 1987. Chromatin higher-order structure studied by neutron scattering and scanning transmission electron microscopy. Proceedings of the National Academy of Sciences 84:7802-7806

52. Gibcus JH, Mirny LA, Dekker J. 2018. A pathway for mitotic chormosome formation. Science 6135:1-18

53. Grosberg AY, Nechaev S, Shakhnovich E. 1988. The role of topological constraints in the kinetics of collapse of macromolecules. Journal De Physique 49:2095-2100

54. Guelen L, Pagie L, Brasset E, Meuleman W, Faza MB, et al. 2008. Domain organization of human chromosomes revealed by mapping of nuclear lamina interactions. Nature 453:948-951

55. Haarhuis JH, van der Weide RH, Blomen VA, Yáñez-Cuna JO, Amendola M, et al. 2017. The Cohesin Release Factor WAPL Restricts Chromatin Loop Extension. Cell 169:693-707.e14

56. Hansen AS, Pustova I, Cattoglio C, Tjian R, Darzacq X. 2017. CTCF and cohesin regulate chromatin loop stability with distinct dynamics. eLife 6:1-33

57. Hansen JC, Connolly M, McDonald CJ, Pan A, Pryamkova A, et al. 2018. The 10-nm chromatin fiber and its relationship to interphase chromosome organization. Biochemical Society transactions 46:67-76

58. Herbert S, Brion A, Arbona JM, Lelek M, Veillet A, et al. 2017. Chromatin stiffening underlies enhanced locus mobility after dna damage in budding yeast. The EMBO journal :e201695842

59. Hyman AA, Weber CA, Jülicher F. 2014. Liquid-Liquid Phase Separation in Biology. Annual Review of Cell and Developmental Biology 30:39-58

60. Kakui Y, Rabinowitz A, Barry DJ, Uhlmann F. 2017. Condensin-mediated remodeling of the mitotic chromatin landscape in fission yeast. Nature Genetics 49:1-11

61. Kalhor R, Tjong H, Jayathilaka N, Alber F, Chen L. 2012. Genome architectures revealed by tethered chromosome conformation capture and population-based modeling. Nature biotechnology 30:90-8

62. Ke Y, Xu Y, Chen X, Feng S, Liu Z, et al. 2017. 3D Chromatin Structures of Mature Gametes and Structural Reprogramming during Mammalian Embryogenesis. Cell 170:367-381.e20

63. Kornberg RD, Stryer L. 1988. Statistical distributions of nucleosomes: nonrandom locations by a stochastic mechanism. Nucleic Acids Research 16:6677-6690

64. Kruithof M, Chien FT, Routh A, Logie C, Rhodes D, van Noort J. 2009. Single-molecule force spectroscopy reveals a highly compliant helical folding for the 30-nm chromatin fiber. Nat. Struct. Mol. Biol. 16:534-540

65. Kwon I, Kato M, Xiang S, Wu L, Theodoropoulos P, et al. 2013. Phosphorylation-regulated Binding of RNA Polymerase II to Fibrous Polymers of Low Complexity Domains. Cell 155:1049-1060

66. Larson AG, Elnatan D, Keenen MM, Trnka MJ, Johnston JB, et al. 2017. Liquid droplet formation by HP1 $\alpha$ suggests a role for phase separation in heterochromatin. Nature 547:236240

67. Lazar-stefanita L, Scolari VF, Mercy G, Muller H, Guérin TM, et al. 2017. Cohesins and condensins orchestrate the $4 \mathrm{D}$ dynamics of yeast chromosomes during the cell cycle. The EMBO Journal 36:2684-2697

68. Lieberman-aiden E, Berkum NLV, Williams L, Imakaev M, Ragoczy T, et al. 2009. Comprehensive Mapping of Long-Range Interactions Reveals Folding Principles of the Human Genome. Science 326:289-294 
69. Lim B, Heist T, Levine M, Fukaya T. 2018. Visualization of Transvection in Living Drosophila Embryos. Molecular Cell 70:287-296

70. Lupiáñez DG, Kraft K, Heinrich V, Krawitz P, Brancati F, et al. 2015. Disruptions of topological chromatin domains cause pathogenic rewiring of gene-enhancer interactions. Cell 161:10121025

71. Mack AH, Schlingman DJ, Ilagan RP, Regan L, Mochrie SGJ. 2012. Kinetics and Thermodynamics of Phenotype: Unwinding and Rewinding the Nucleosome. Journal of Molecular Biology 423:687-701

72. Matera AG. 1999. Nuclear bodies: multifaceted subdomains of the interchromatin space. Trends in cell biology 9:302-309

73. Merkenschlager M, Nora EP. 2016. Ctcf and cohesin in genome folding and transcriptional gene regulation. Annual review of genomics and human genetics 17:17-43

74. Murayama Y, Samora CP, Kurokawa Y, Iwasaki H, Uhlmann F. 2018. Establishment of DNADNA Interactions by the Cohesin Ring. Cell 172:1-13

75. Murayama Y, Uhlmann F. 2014. Biochemical reconstitution of topological DNA binding by the cohesin ring. Nature 505:367-371

76. Murayama Y, Uhlmann F. 2015. DNA Entry into and Exit out of the Cohesin Ring by an Interlocking Gate Mechanism. Cell 163:1628-1640

77. Nagano T, Lubling Y, Stevens TJ, Schoenfelder S, Yaffe E, et al. 2013. Single-cell Hi-C reveals cell-to-cell variability in chromosome structure. Nature 502:59-64

78. Nagano T, Lubling Y, Várnai C, Dudley C, Leung W, et al. 2017. Cell-cycle dynamics of chromosomal organization at single-cell resolution. Nature 547:61-67

79. Narlikar GJ, Sundaramoorthy R, Owen-Hughes T. 2013. Mechanisms and functions of ATPdependent chromatin-remodeling enzymes. Cell 154:490-503

80. Nasmyth K. 2001. Disseminating the Genome: Joining, Resolving, and Separating Sister Chromatids During Mitosis and Meiosis. Annual Review of Genetics 35:673-745

81. Naumova N, Imakaev M, Fudenberg G, Zhan Y, Lajoie BR, et al. 2013. Organization of the mitotic chromosome. Science 342:948-53

82. Nikiforova MN, Stringer JR, Blough R, Medvedovic M, Fagin JA, Nikiforov YE. 2000. Proximity of chromosomal loci that participate in radiation-induced rearrangements in human cells. Science 290:138-141

83. Nora EP, Goloborodko A, Valton AL, Gibcus JH, Uebersohn A, et al. 2017. Targeted Degradation of CTCF Decouples Local Insulation of Chromosome Domains from Genomic Compartmentalization. Cell 169:930-944.e22

84. Nora EP, Lajoie BR, Schulz EG, Giorgetti L, Okamoto I, et al. 2012. Spatial partitioning of the regulatory landscape of the X-inactivation centre. Nature 485:381-385

85. Nozaki T, Imai R, Tanbo M, Nagashima R, Tamura S, Tani T. 2017. Dynamic Organization of Chromatin Domains Revealed by Super-Resolution Live-Cell Imaging. Molecular Cell 67:1-12

86. Nuebler J, Fudenberg G, Imakaev M, Abdennur N, Mirny LA. 2018. Chromatin organization by an interplay of loop extrusion and compartmental segregation. Proceedings of the National Academy of Sciences 115:E6697-E6706

87. Ou HD, Phan S, Deerinck TJ, Thor A, Ellisman MH, O'Shea CC. 2017. ChromEMT: Visualizing 3D chromatin structure and compaction in interphase and mitotic cells. Science 357:1-13

88. Padinhateeri R, Marko JF. 2011. Nucleosome positioning in a model of active chromatin remodeling enzymes. Proceedings of the National Academy of Sciences of the United States of America 108:7799-7803

89. Parmar JJ, Marko JF, Padinhateeri R. 2014. Nucleosome positioning and kinetics near transcription-start-site barriers are controlled by interplay between active remodeling and DNA sequence. Nucleic Acids Research 42:128-36

90. Patel A, Malinovska L, Saha S, Wang J, Alberti S, et al. 2017. Biochemistry: ATP as a biological hydrotrope. Science 356:753-756 
91. Patrick H, Laroche T, Shimada K, Furrer P, Gasser SM. 2001. Chromosome Dynamics in the Yeast Interphase Nucleus. Science 294:2181-2186

92. Pereira MCF, Brackley CA, Michieletto D, Annunziatella C, Bianco S, et al. 2018. Complementary chromosome folding by transcription factors and cohesin. bioRxiv

93. Pope BD, Ryba T, Dileep V, Yue F, Wu W, et al. 2014. Topologically associating domains are stable units of replication-timing regulation. Nature 515:402-405

94. Quinodoz SA, Ollikainen N, Tabak B, Palla A, Schmidt JM, et al. 2018. Higher-Order Interchromosomal Hubs Shape 3D Genome Organization in the Nucleus. Cell 174:1-14

95. Racko D, Benedetti F, Dorier J, Stasiak A. 2017. Transcription-induced supercoiling as the driving force of chromatin loop extrusion during formation of TADs in interphase chromosomes. Nucleic Acids Research 46:1648-1660

96. Ramani V, Deng X, Qiu R, Gunderson KL, Steemers FJ, et al. 2017. Massively multiplex single-cell Hi-C. Nature Methods 14:263-266

97. Rao SS, Huang SC, Hilaire BGS, Engreitz JM, Perez EM, et al. 2017. Cohesin Loss Eliminates All Loop Domains. Cell 171:305-320.e24

98. Rao SSP, Huntley MH, Durand NC, Stamenova EK, Bochkov ID, et al. 2014. A 3D Map of the Human Genome at Kilobase Resolution Reveals Principles of Chromatin Looping. Cell 159:1665-1680

99. Razin SV, Gavrilov AA. 2014. Chromatin without the 30-nm fiber. Epigenetics 9:653-657

100. Reddy KL, Zullo JM, Bertolino E, Singh H. 2008. Transcriptional repression mediated by repositioning of genes to the nuclear lamina. Nature 452:243-247

101. Ricci MA, Manzo C, García-Parajo MF, Lakadamyali M, Cosma MP. 2015. Chromatin fibers are formed by heterogeneous groups of nucleosomes in vivo. Cell 160:1145-1158

102. Robinson PJJ, Fairall L, Huynh VAT, Rhodes D. 2006. EM measurements define the dimensions of the "30-nm" chromatin fiber: Evidence for a compact, interdigitated structure. Proceedings of the National Academy of Sciences 103:6506-6511

103. Rosa A, Everaers R. 2008. Structure and dynamics of interphase chromosomes. PLoS Computational Biology 4:1-10

104. Rosa A, Zimmer C. 2014. Computational models of large-scale genome architecture. In International review of cell and molecular biology, vol. 307. Elsevier, 275-349

105. Rubinstein M, Colby R. 2003. Polymer Physics. OUP Oxford

106. Sanborn AL, Rao SSP, Huang SC, Durand NC, Huntley MH, et al. 2015. Chromatin extrusion explains key features of loop and domain formation in wild-type and engineered genomes. Proceedings of the National Academy of Sciences 112:201518552

107. Sazer S, Schiessel H. 2018. The biology and polymer physics underlying large-scale chromosome organization. Traffic 19:87-104

108. Schalbetter SA, Goloborodko A, Fudenberg G, Belton JM, Miles C, et al. 2017. SMC complexes differentially compact mitotic chromosomes according to genomic context. Nature Cell Biology 19:1-12

109. Schalch T, Duda S, Sargent DF, Richmond TJ. 2005. X-ray structure of a tetranucleosome and its implications for the chromatin fibre. Nature 436:138-141

110. Schwarzer W, Abdennur N, Goloborodko A, Pekowska A, Fudenberg G, et al. 2017. Two independent modes of chromatin organization revealed by cohesin removal. Nature 551:51-56

111. Solovei I, Kreysing M, Lanctôt C, Kösem S, Peichl L, et al. 2009. Nuclear Architecture of Rod Photoreceptor Cells Adapts to Vision in Mammalian Evolution. Cell 137:356-368

112. Song F, Chen P, Sun D, Wang M, Dong L, et al. 2014. Cryo-EM Study of the Chromatin Fiber Reveals a Double Helix Twisted by Tetranucleosomal Units. Science 344:376-380

113. Strom AR, Emelyanov AV, Mir M, Fyodorov DV, Darzacq X, Karpen GH. 2017. Phase separation drives heterochromatin domain formation. Nature 547:241-245

114. Szabo Q, Jost D, Chang JM, Cattoni DI, Papadopoulos GL, et al. 2018. TADs are 3D structural units of higher-order chromosome organization in Drosophila. Science Advances 4:1-13 
115. Tedeschi A, Wutz G, Huet S, Jaritz M, Wuensche A, et al. 2013. Wapl is an essential regulator of chromatin structure and chromosome segregation. Nature 501:564-568

116. Teves SS, An L, Hansen AS, Xie L, Darzacq X, Tjian R. 2016. A dynamic mode of mitotic bookmarking by transcription factors. eLife 5:1-24

117. Therizols P, Duong T, Dujon B, Zimmer C, Fabre E. 2010. Chromosome arm length and nuclear constraints determine the dynamic relationship of yeast subtelomeres. Proceedings of the National Academy of Sciences 107:2025-30

118. Therizols P, Illingworth RS, Courilleau C, Boyle S, Wood AJ, Bickmore WA. 2014. Chromatin decondensation is sufficient to alter nuclear organization in embryonic stem cells. Science 346:1238-1242

119. Ulianov S, Razin S, Shevelyov Y. 2015. Active chromatin and transcription play a key role in chromosome partitioning into TADs. Genome research :70-84

120. van Steensel B, Belmont AS. 2017. Lamina-associated domains: links with chromosome architecture, heterochromatin, and gene repression. Cell 169:780-791

121. Vian L, Pekowska A, Rao SS, Kieffer-Kwon KR, Jung S, et al. 2018. The Energetics and Physiological Impact of Cohesin Extrusion. Cell 173:1165 - 1178.e20

122. Walther N, Hossain MJ, Politi AZ, Koch B, Kueblbeck M, et al. 2018. A quantitative map of human Condensins provides new insights into mitotic chromosome architecture. The Journal of Cell Biology 217:2309-2328

123. Wang S, Su Jh, Beliveau BJ, Bintu B, Moffitt JR, et al. 2016. Spatial organization of chromatin domains and compartments in single chromosomes. Science 353:598-602

124. Widom J, Klug A. 1985. Structure of the $3000 \AA$ chromatin filament: X-ray diffraction from oriented samples. Cell 43:207-213

125. Wiggins PA, van der Heijden T, Moreno-Herrero F, Spakowitz A, Phillips R, et al. 2006. High flexibility of DNA on short length scales probed by atomic force microscopy. Nature nanotechnology 1:137-41

126. Wong H, Marie-Nelly H, Herbert S, Carrivain P, Blanc H, et al. 2012. A predictive computational model of the dynamic 3D interphase yeast nucleus. Current Biology 22:1881-1890

127. Woringer M, Darzacq X. 2018. Protein motion in the nucleus: from anomalous diffusion to weak interactions. arXiv :1-11

128. Wutz G, Várnai C, Nagasaka K, Cisneros DA, Stocsits RR, et al. 2017. Topologically associating domains and chromatin loops depend on cohesin and are regulated by CTCF, WAPL, and PDS5 proteins. The EMBO Journal 36:e201798004

129. Xiang W, Roberti MJ, Hériché JK, Huet S, Alexander S, Ellenberg J. 2018. Correlative live and super-resolution imaging reveals the dynamic structure of replication domains. The Journal of Cell Biology 217:1973-1984

130. Yan J, Maresca TJ, Skoko D, Adams CD, Xiao B, et al. 2007. Micromanipulation Studies of Chromatin Fibers in Xenopus Egg Extracts Reveal ATP-dependent Chromatin Assembly Dynamics. Mol. Biol. Cell 18:464-474

131. Zhan Y, Mariani L, Barozzi I, Schulz EG, Blüthgen N, et al. 2017. Reciprocal insulation analysis of Hi-C data shows that TADs represent a functionally but not structurally privileged scale in the hierarchical folding of chromosomes. Genome Research 27:479-490

132. Zhang Z, Wippo CJ, Wal M, Ward E, Korber P, Pugh BF. 2011. A packing mechanism for nucleosome organization reconstituted across a eukaryotic genome. Science 332:977-980

133. Zhu L, Brangwynne CP. 2015. Nuclear bodies: the emerging biophysics of nucleoplasmic phases. Current opinion in cell biology 34:23-30

134. Zuin J, Franke V, van IJcken WF, van der Sloot A, Krantz ID, et al. 2014. A CohesinIndependent Role for NIPBL at Promoters Provides Insights in CdLS. PLoS Genetics 10:1-15 